\section{iPS models of neural development}

The cellular basis of neurodevelopmental disorders, such as autism, is not well understood, and it is important to establish in vitro models that recapitulate neural development in humans. Now, Flora Vaccarino and colleagues report the development of multilayered structures from human induced pluripotent stem cell lines (hiPSCs) that harbor hallmarks of the embryonic telencephalon (Proc. Natl. Acad. Sci. USA, published online 3 July 2012; doi: 10.1073/pnas.1202944109). Methods to culture cortical tissues from embryonic stem cells have been reported, and the authors applied these protocols to hiPSCs. The resulting three-dimensional structures included neural tube-like substructures, radial glia-like progenitors and more mature neurons. After the cultures had been differentiated 70 days in vitro, the authors observed immature synaptic boutons and immature synapses. Analysis of the transcriptomes of cultures at day $\mathbf{5 0}$ identified thousands of genes that were differentially expressed relative to undifferentiated hiPSCs, and pathway analyses suggested enrichment for neural development genes. The transcriptome at day $\mathbf{5 0}$ was more typical of dorsal, rather than ventral, forebrain. Comparisons to human dorsal forebrain at $\mathbf{1 5}$ different time points showed that the transcriptome at day 50 had the best correlation with cerebral cortex at 4-10 weeks postconception.

\section{Transgenerational epigenetic inheritance and piRNAs}

Silencing of multicopy transgenes is thought to occur via production of double-stranded RNAs, which are processed by Dicer to small interfering RNAs (siRNAs) that are loaded onto Argonaute proteins to target transcriptional or post-transcriptional silencing. Now, Craig Mello and colleagues and Shawn Ahmed, Eric Miska and colleagues separately report that piwi-interacting RNAs (piRNAs) can initiate stable, heritable epigenetic silencing in the germline of Caenorhabditis elegans (Cell 150, 65-77, 2012, and Cell 150, 88-99, 2012). Both groups use singlecopy insertion transgenes to identify a pathway involving chromatinassociated factors and RNA interference (RNAi) components that are required for the maintenance of multigenerational silencing. Both groups find that piRNAs are required for initiation but not maintenance of silencing. This work suggests a new mechanism of transgene silencing that is independent of the production of siRNAs from double-stranded RNA. The authors suggest that piRNAs may scan the genome for foreign sequences and initiate targeted silencing.

\section{CXCL1 and CXCL2 link metastasis and chemoresistance}

Metastasis and resistance to chemotherapy co-occur in cancer, although the molecular links between them are not understood. Previous data suggested that increased expression of CXCL1 and CXCL2 has an important role in breast cancer metastasis, and Joan Massagué and colleagues now report that these chemokines mediate a signaling network that links lung metastasis and chemotherapy resistance in breast cancer (Cell 150, 165-178, 2012). The authors knocked down CXCL1 and CXCL2 in a

Written by Orli Bahcall, Pamela Feliciano, Emily Niemitz \& Kyle Vogan metastatic lung cancer xenograft model and analyzed the tumor microenvironment, finding that there were fewer $\mathrm{CD} 11 \mathrm{~b}^{+} \mathrm{Gr}^{+}$myeloid cells than in controls. Hypothesizing that these cells might secrete paracrine factors that augment cancer cell survival, the authors found that expression of the paracrine factors S100A8 and S100A9 correlates with that of CXCL1 in breast cancer. Mammary tumor growth and lung metastasis were decreased in S100a9-/- mice, suggesting that S100A8 and S100A9 are required for tumor cell survival and metastasis. The authors analyzed breast tumors before and after chemotherapy and observed an increase in S100A9 after treatment. Chemotherapy also induces tumor necrosis factor (TNF)- $\alpha$ in stromal cells, which further amplifies the CXCL1/ CXCL2-S100A8/S100A9 pathway in tumor cells. Preclinical work in mouse models showed that chemotherapy combined with treatment with a CXCR2 antagonist might be an effective way to reduce tumor growth.

\section{Light-green tomatoes}

Modern tomato breeding practices have selected for fruit with a uniform light-green color before ripening, a trait that promotes even ripening at the cost of reducing fruit sugar content. This trait, which is determined by the quantity and distribution of chlorophyll in the unripe fruit, is controlled by a single recessive locus uniform ripening $(u)$. Ann Powell and colleagues (Science 336, 1711-1715, 2012) now show that the $u / u$ trait is caused by a loss-of-function mutation in SlGLK2, which encodes a transcription factor known to regulate chloroplast formation. The authors mapped the $u$ locus to a 60 -kb region on chromosome 12 . They then sequenced SlGLK2, the top candidate gene in the region, and identified a 1 -bp insertion shared by all $u / u$ varieties that results in a frameshift and premature termination of translation. Co-suppression of SlGLK2 in $U / U$ plants produced unripe fruit that was uniformly light green. Conversely, expression of Arabidopsis thaliana GLK2 in $u / u$ tomato fruit before ripening produced unripe fruit that was uniformly dark green and whose sugar content was increased by $40 \%$ after ripening. Application of these findings could lead to improvements in tomato fruit quality and production traits.

$K V$

\section{Regulatory changes in primates}

Gregory Crawford and colleagues take a useful approach to consider how regulatory elements contribute to gene expression differences and evolution between primate species (PLoS Genet. 8, e1002789, 2012). The authors report the results of genome-wide DNase-seq in primary skin fibroblasts and lymphoblastoid cell lines from humans, chimpanzees and rhesus macaques, comparing DNase I-hypersensitive (DHS) sites that mark regulatory elements. Whereas most DHS sites are conserved, the authors were able to identify species-specific differences. They further identified several hundred DHS sites that were gained or lost in the human and chimpanzee lineages relative to macaques, as well as a set of common DHS regions shared across species. They compared their data to DNase-seq data sets for $\mathbf{2 7}$ human cell types in ENCODE and found that most of the human DHS gains were cell type specific, whereas the common DHS sites were found across human cell types. Species-specific DHS sites are enriched near genes showing differential expression in these species. The authors also found evidence for branch-specific selection at DHS sites that were gained in the human or chimpanzee lineages. This work provides insights into the tissue- and species-specific activities of regulatory elements and their role in evolution. 